\title{
VIOLÊNCIA DOMÉSTICA CONTRA AS MULHERES: CONHECIMENTOS, ATITUDES E BARREIRAS DO ENFERMEIRO DE FAMÍLIA
}

\author{
Domestic violence against women: knowledge, attitudes and barriers of family nurses \\ Violencia doméstica contra las mujeres: conocimiento, actitudes y barreras de las enfermeras de familia
}

\author{
Isabel Oliveira, ${ }^{*}$ Cíntia Oliveira, ${ }^{* *}$ Juliana Carvalho, ${ }^{* * *}$ Nuno Santos, ${ }^{* * *}$ Ana Torres ${ }^{* * *}$
}

\section{RESUMO}

Enquadramento: a violência doméstica é um problema de saúde pública. O enfermeiro de família, enquanto contacto privilegiado com mulheres e famílias, pode desempenhar um papel relevante na abordagem à mulher vítima de violência. Objetivo: identificar os conhecimentos, atitudes e barreiras dos enfermeiros de família na abordagem às mulheres vítimas de violência doméstica. Metodologia: desenho quantitativo, exploratório, descritivo e transversal, numa amostra não probabilística por conveniência, constituída por cinquenta enfermeiros de família. O instrumento de recolha de dados foi um questionário de autopreenchimento. No tratamento recorreu-se aos aplicativos QDA Miner 4 Lite e IBM SPSS Statistic, versão 21.0. Resultados: dos participantes, 78\% refere não ter tido formação sobre violência doméstica. Evidenciam falta de conhecimento, reconhecendo, no entanto, que a identificação e encaminhamento das vítimas é responsabilidade dos profissionais de saúde. Apontam como principais barreiras a falta de protocolos de atuação e de regulamentação que legitime a sua intervenção e, em menor percentagem, a falta de conhecimento/formação para a abordagem à vítima. Conclusão: torna-se assim necessário dimensionar as necessidades ao nível da formação pré e pós-graduada, infundindo nos currículos esta temática e capacitando assim atuais e futuros profissionais na abordagem à mulher vítima de violência doméstica.

Palavras-chave: violência doméstica; enfermeiro de família; conhecimentos; atitude

*RN, Professora Adjunta. Enfermeira Especialista em Enfermagem de Reabilitação

https://orcid.org/0000-0001-6627-3907

autor de correspondência:

isabel.oliveira@essnortecvp.pt

${ }^{* *}$ RN, Enfermeira, Centro Internacional de Medicina Integrativa

${ }^{* * *}$ RN, Enfermeiro, Centro Hospitalar de Entre Douro e Vouga

${ }^{* * * * P h D}$, Professora Adjunta da Escola Superior de Saúde Norte da Cruz Vermelha Portuguesa/ CINTESIS

https://orcid.org/0000-0002-8919-0378

Como referenciar:

Oliveira, I., Oliveira, C., Carvalho, J., Santos, N., \& Torres, A. (2020). Violência doméstica contra as mulheres: conhecimentos, atitudes e barreiras do enfermeiro de família. Revista de Investigação \& Inovação em Saúde, vol.3(2). 29-38. doi.org/10.37914/riis.v3i2.102

\section{ABSTRACT}

Background: domestic violence is a public health problem. Family nurses, as privileged contact with women and families, can play an important role in the prevention and approach to women victims of violence. Objective: identify knowledge, attitudes and barriers of family nurses in the approach of women victims of domestic violence. Methodology: quantitative, exploratory, descriptive and cross-sectional design, in a non-probabilistic sample for convenience, consisting of fifty family nurses. The data collection instrument was a self-administered questionnaire. For data analysis, software QDA Miner 4 Lite and IBM SPSS Statistic, version 21.0, were used. Results: of the participants, $78 \%$ report having no training on domestic violence and report lack of knowledge about it, recognizing, however, that the identification and referral of victims is the responsibility of health professionals. They point out as main barriers the lack of action and regulation protocols that legitimize their intervention and, to a lesser extent, the lack of knowledge / training to approach the victim. Conclusion: it is therefore necessary to measure the needs at the level of pre- and post-graduate training, infusing this theme in the curricula and thus training current and future professionals in the identification and approach to women victims of domestic violence.

Keywords: domestic violence; family nurse; knowledge; attitude

\section{RESUMEN}

Marco Contextual: la violencia doméstica es un problema de salud pública. La enfermera de familia, como contacto privilegiado con la mujer y la familia, puede jugar un papel importante en el abordaje de las mujeres víctimas de violencia. Objetivo: Identificar conocimientos, actitudes y barreras de enfermeras de familia en la identificación y derivación de mujeres víctimas de violencia intrafamiliar. Metodología: diseño cuantitativo, exploratorio, descriptivo y transversal, en una muestra no probabilística por conveniencia, con cincuenta enfermeros familiares. El instrumento de recolección de datos fue un cuestionario autoadministrado. En su tratamiento se utilizó el software informático QDA Miner 4 Lite e IBM SPSS Statistic, versión 21.0. Resultados: los participantes, $78 \%$ informa que no tuvo capacitación sobre este tema y evidencia un desconocimiento al respecto, reconociendo, sin embargo, que la identificación y derivación de las víctimas es responsabilidad de los profesionales de la salud. Señalan como principales barreras la falta de protocolos de actuación y regulación que legitimen su intervención y, en un menor porcentaje, la falta de conocimiento / formación para acercarse a la víctima. Conclusión: es necesario medir las necesidades en materia de formación de pre y posgrado, infundiendo esta temática en los planes de estudio y formando así a los actuales y futuros profesionales en la identificación y abordaje de las mujeres víctimas de violencia intrafamiliar.

Palabras clave: violencia doméstica; enfermero familiar; conocimiento; actitudes
Recebido para publicação em: $3 / 11 / 2020$ Aceite para publicação em: 12/12/2020 
Violência doméstica contra as mulheres: conhecimentos, atitudes e barreiras do enfermeiro de família

\section{INTRODUÇÃO}

A violência contra as mulheres é um crime com consequências graves para a saúde da mulher, nomeadamente ao nível físico, mental, sexual e reprodutivo, tendo por isso repercussões em toda a estrutura familiar e comunidade (Organização Mundial da Saúde [OMS], 2020). Neste contexto, o enfermeiro de família "assume a responsabilidade pela prestação de cuidados de enfermagem globais a famílias, em todas as fases da vida e em todos os contextos da comunidade" (Decreto Lei n.ㅇ 118/2014, de 5 de agosto, p. 4070), podendo desempenhar um papel relevante, na abordagem mulher vítima de violência doméstica. Face à pertinência da temática e considerando que não foi encontrada, em Portugal, evidência sobre os conhecimentos e atitudes dos enfermeiros de família neste contexto, torna-se relevante identificá-los, assim como identificar as barreiras na abordagem destas mulheres. Pretende-se assim encontrar estratégias de melhoria na abordagem às mulheres vítimas de violência doméstica no contexto dos cuidados de saúde Primários (CSP), considerando que estes se constituem como ponto privilegiado de acesso aos serviços de saúde e prevenção primária e secundária.

\section{ENQUADRAMENTO/FUNDAMENTAÇÃO TEÓRICA}

A violência contra a mulher é reconhecida pela OMS como um grave problema mundial e uma questão de saúde pública (World Health Organization [WHO], 2017). É altamente prevalente, sendo que a violência praticada pelo parceiro íntimo é a forma mais comum (OMS, 2020). Em Portugal, a violência doméstica é tipificada como crime público através da Lei no 59/2007, de 4 de setembro e especificada no artigo
152 do Código Penal (Lei n. $\cong 59 / 2007$, de 4 de setembro). No ano de 2019, a Associação Portuguesa de Apoio à Vítima (APAV) registou um total de 11.676 vítimas de crime. Cerca de $80 \%$ destas eram do sexo feminino, sendo que as faixas etárias mais frequentes se situavam entre os 25 e os 54 anos de idade. As relações entre autor e vítima de crime, eram relações de intimidade. É prevalente o tipo de vitimização continuada, sendo os locais mais referenciados para a ocorrência da violência a residência comum de vítima e perpetrador (APAV, 2020).

Face aos desafios desta problemática têm vindo a ser implementados em Portugal sucessivos planos de prevenção e combate, estando atualmente em vigor o quinto Plano Nacional de Prevenção e Combate à Violência Doméstica e de Género 2014-2017 (V PNPCVDG). Este assenta nos pressupostos da Convenção de Istambul e assume-se como uma mudança de paradigma nas políticas públicas nacionais de combate a todas estas formas de violação dos direitos humanos fundamentais, como são os vários tipos de violência de género, incluindo a violência doméstica. Encontra-se estruturado em cinco áreas estratégicas: a primeira incide na prevenção, sensibilização e educação; a segunda, na proteção das vítimas e proteção da sua integração; a terceira, na intervenção junto dos agressores; a quarta, na formação e qualificação dos profissionais; por fim, a quinta área estratégica incide na investigação e monitorização (Presidência do Conselho de Ministros no 103/2013, 2013).

O enfermeiro de família, no contexto da problemática da violência doméstica contra a mulher, detém um conjunto de competências que vão ao encontro e permitem dar resposta às áreas estratégicas definidas 
Violência doméstica contra as mulheres: conhecimentos, atitudes e barreiras do enfermeiro de família

no V PNPCVDG, nomeadamente ao nível da prevenção e controlo de situações complexas e na promoção e recuperação do bem-estar da família em situações complexas (Regulamento n.ㅇ 428/2018, de 16 de julho). Além disso, os serviços de saúde primários têm sido escolhidos pelas mulheres para relatar as situações de violência em que vivem (Rodrigues et al., 2018). Para que o enfermeiro de família possa intervir assertivamente deve reunir conhecimentos e atitudes, que fundamentem o seu processo de tomada de decisão. Segundo a Classificação Internacional para a Prática de Enfermagem (CIPE $\left.{ }^{\circledR}\right)$, o conhecimento é um "conteúdo específico de ensinamento baseado na sabedoria adquirida, na informação apreendida ou competência" (Conselho Internacional dos Enfermeiros, 2016, p. 47) e a atitude define-se como "um processo psicológico de modelos mentais e opiniões" (Conselho Internacional dos Enfermeiros, 2016, p. 41). Desta forma, e existindo evidência que refere parte por dos enfermeiros uma baixa taxa de encaminhamento destas mulheres em distintos contextos de prática clínica, nomeadamente em serviços de urgência (Cho, Cha \& Yoo, 2015) por falta de conhecimento, levantou-se a seguinte questão de investigação: Quais os conhecimentos e atitudes do enfermeiro de família na abordagem à mulher vítima de violência doméstica?

Os resultados deste estudo contribuirão para explorar a realidade portuguesa ao nível dos enfermeiros de família e orientar para estratégias que potenciem a melhoria dos cuidados prestados a estas mulheres e suas famílias ao nível dos CSP.

\section{METODOLOGIA}

Para responder à questão de investigação, foi desenvolvido um estudo quantitativo do tipo exploratório, descritivo e transversal, com uma amostra não probabilística de conveniência. Foram definidos como critérios de inclusão ser enfermeiro de família em exercício de funções em unidades de saúde familiar ou unidades de cuidados de saúde personalizados; que se desloquem à instituição de ensino superior para realização de formação pósgraduada, formação contínua ou participação em eventos científicos e que consintam à participação. Para a recolha de dados foi utilizado um questionário intitulado "Violência contra la mujer por parte de su pareja: opinión del personal médico del IMSS Morelos", desenvolvido por Méndez-Hernández, ValdezSantiago, Viniegra-Velázquez, Rivera-Rivera \& Salmerón-Castro (2003) num estudo que avalia os conhecimentos, atitudes e barreiras do pessoal médico do Instituto Mexicano del Seguro Social do estado de Morelos e cujo processo de tradução e adaptação cultural foi realizado de acordo com o proposto por Wild et al. (2005). A sua tradução foi realizada por dois tradutores nativos em português e consensualizada por um terceiro elemento (investigador do estudo). A retro-tradução foi realizada por um tradutor nativo de língua castelhana e posteriormente enviada ao autor que a validou. A versão final do questionário foi submetida a um processo de equivalência cognitiva através da sua aplicação a cinco enfermeiros de família, que cumpriam os critérios de inclusão no estudo, garantindo-se a adequação do mesmo aos seus utilizadores. Relativamente à estruturação, o questionário encontra-se organizado em seis partes, a primeira parte refere-se à informação 


\section{Violência doméstica contra as mulheres: conhecimentos, atitudes e barreiras do enfermeiro de família}

sociodemográfica; a segunda parte, dá a conhecer o nível de instrução prévia dos enfermeiros de família sobre a violência contra a mulher; a terceira parte, expõe a atitude cognitiva dos enfermeiros de família sobre o tema enquadrando o seu conhecimento; na quarta parte, é possível identificar os fatores institucionais e da prática profissional que os enfermeiros de família percebem como barreiras na identificação das mulheres agredidas que chegam à consulta, todas elas constituídas por questões de resposta dicotómica (sim/não), perguntas de escolha múltipla e perguntas de resposta aberta. Na quinta parte, apresenta um total de quarenta afirmações sobre as quais se solicita aos participantes que expressem a sua concordância, através de uma escala de Likert, sobre a sua atitude afetiva no que diz respeito ao interesse em identificar e encaminhar os casos de mulheres agredidas, bem como o interesse na capacitação sobre o tema. A sexta parte, constituída por questões de resposta de escolha múltipla e resposta aberta, permite conhecer a atitude no que respeita à conduta dos profissionais diante dos casos de mulheres agredidas, em contexto laboral. Para o tratamento de dados recorreu-se aos programas informáticos QDA Miner 4 Lite e IBM SPSS Statistics, versão 21.0. O estudo obteve o parecer favorável da Comissão de Ética da instituição de ensino superior onde o estudo decorreu.

\section{RESULTADOS}

A recolha de dados foi realizada entre julho de 2019 a janeiro de 2020 tendo sido entregues 100 questionários dos quais cinquenta foram devolvidos e analisados. Participaram neste estudo 50 enfermeiros, $88 \%$ do sexo feminino $(n=44)$, com uma idade média

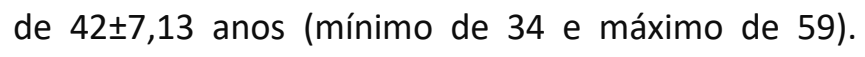
Relativamente ao estado civil, 56\% ( $n=28)$ eram casados ou a viver em união de facto, $24 \%$ ( $n=12)$ eram solteiros e $20 \%$ ( $n=10)$ eram divorciados, separados ou viúvos. Dos participantes, $66 \% \quad(n=33)$ eram enfermeiros de cuidados gerais e $34 \%(n=17)$ com título profissional de enfermeiro especialista. Quanto à formação académica, 36\% ( $n=18)$ eram licenciados, 44\% ( $n=22)$ tinham formação pós-graduada não conferente de grau e $20 \%(n=10)$ eram mestres. Relativamente ao tempo de exercício, a média de tempo de serviço dos participantes foi de $19,1 \pm 6,4$ anos (mínimo de 12 e máximo de 36 anos).

\section{Formação prévia}

No que concerne a formação prévia, 78\% (n=39) dos participantes afirmam nunca ter tido formação nesta área, $60 \%(n=30)$ nunca recebeu apoio para fazer formação por parte da sua entidade patronal, sendo que $40 \%(n=20)$ deles identificam como razão para a falta de apoio a ausência de formação interna sobre este tema, a falta de financiamento para a sua realização e a falta de interesse no tema por parte das instituições onde exercem funções.

\section{Atitudes cognitivas (Conhecimento)}

As atitudes cognitivas referem-se ao conhecimento que os enfermeiros de família têm sobre o tema da violência doméstica. Neste domínio, $88 \%(n=44)$ dos enfermeiros de família referiram conhecer a definição de violência doméstica e 100 \% ( $n=50)$ são da opinião que esta pode acontecer em qualquer contexto socioeconómico e educativo. Dos participantes, $64 \%$ $(n=32)$ consideram que o motivo do homem praticar a violência doméstica é o facto de este ter presenciado situações de violência na infância. Sobre a média 
Violência doméstica contra as mulheres: conhecimentos, atitudes e barreiras do enfermeiro de família

aproximada dos casos de violência em Portugal, 22\% $(n=11)$ afirmaram desconhecer.

Foi ainda solicitado aos participantes em questão aberta que identificassem separadamente os danos físicos, caraterísticas psicológicas e danos sexuais (tabela 1) que já observaram ou que pensem ser observados em mulheres vítimas de violência doméstica. A frequência dos dados elucida sobre a relevância dada pelos participantes, em cada uma das dimensões, aos aspetos específicos de cada uma delas.

Tabela 1

Danos físicos, caraterísticas psicológicas e danos sexuais que participantes observaram em mulheres vítimas de violência doméstica

\begin{tabular}{|c|c|c|}
\hline Danos Físicos & $\mathbf{n}$ & $\%$ \\
\hline Hematomas & 35 & 43,75 \\
\hline Fraturas & 25 & 31,25 \\
\hline Feridas & 14 & 17,5 \\
\hline Queimadura & 3 & 3,75 \\
\hline Outras lesões corporais & 3 & 3,75 \\
\hline Total & 80 & 100 \\
\hline \multicolumn{3}{|c|}{ Caraterísticas Psicológicas } \\
\hline Baixa Autoestima & 21 & 21,43 \\
\hline Medo & 21 & 21,43 \\
\hline Depressão & 18 & 18,37 \\
\hline Ansiedade & 12 & 12,24 \\
\hline Tristeza & 6 & 6,12 \\
\hline Submissão & 5 & 5,1 \\
\hline Insegurança & 4 & 5,08 \\
\hline Isolamento & 4 & 4,08 \\
\hline Vergonha & 3 & 3,06 \\
\hline Labilidade Emocional & 3 & 3,06 \\
\hline Insónia & 1 & 1,02 \\
\hline Total & 80 & 100 \\
\hline \multicolumn{3}{|l|}{ Danos sexuais } \\
\hline Lesões genitais & 15 & 29,41 \\
\hline Lesões vaginais & 13 & 25,49 \\
\hline Diminuição da libido & 11 & 7,84 \\
\hline Lesões anais & 4 & 5,88 \\
\hline Dispareunia & 3 & 3,92 \\
\hline Dor genital & 2 & 3,92 \\
\hline Repulsa sexual & 2 & 3,92 \\
\hline $\begin{array}{l}\text { Doenças sexualmente } \\
\text { transmissíveis }\end{array}$ & 1 & 1,96 \\
\hline Total & 51 & 100 \\
\hline
\end{tabular}


A maior parte dos participantes $(78 \%, n=39)$ referiu ainda conhecer alguma instituição para onde pudessem referenciar as vítimas de violência, bem como $68 \%(n=34)$ referiu conhecer as leis portuguesas que protegem as mulheres que sofrem violência por parte do parceiro. As instituições mais mencionadas foram a APAV, por $52 \%(n=26)$ dos participantes, seguida da Guarda Nacional Republicana, com 10\%, $(n=5)$ e $8 \%(n=4)$ identificaram a Polícia de Segurança Pública. Relativamente ao conhecimento sobre leis que protejam as mulheres que sofrem violência por parte do parceiro, 20\% $(n=10)$ referiram alguns articulados legais, sendo que apenas um participante fez alusão correta à legislação aplicável neste contexto. Quase todos os participantes $(98 \%, n=49)$ consideram que a violência que o parceiro exerce contra a mulher se repercute de maneira muito importante sobre a sua saúde e todos eles $(100 \%, n=50)$ referem que, em situações extremas, pode até ocasionar a morte da vítima ou o suicídio.

\section{Atitudes afetivas}

As atitudes afetivas referem-se ao interesse dos enfermeiros de família na identificação e encaminhamento das mulheres, vítimas de violência doméstica, bem como na sua capacitação sobre o tema. Relativamente ao interesse em identificar estas mulheres, $96 \%(n=48)$ dos participantes partilharam da opinião que seria útil identificar os casos de mulheres agredidas durante a sua consulta e $86 \%(n=43)$ consideraram que detetar os casos de mulheres maltratadas pelos parceiros deve ser uma das tarefas do enfermeiro. Dos participantes, $76 \%(n=38)$ são da opinião que os casos de mulheres agredidas são difíceis de identificar numa consulta de rotina e $76 \%$ $(n=38)$ realizaram perguntas específicas para os identificar apenas quando suspeitaram. Para 66\% $(n=33)$ dos participantes compete ao enfermeiro de família ter a iniciativa de, junto da mulher, averiguar as questões relacionadas com a violência. Quanto ao tipo de violência, $64 \%$ ( $n=32$ ) dos participantes referem que a violência psicológica/verbal não é um problema na sua prática clínica, assim como $44 \%(n=22)$ deles referem que os casos de mulheres que sofrem violência sexual por parte de seus parceiros não são um problema frequente, sendo que $44 \%(n=22)$ dos participantes referem ter já identificado casos de mulheres vítimas de violência pelo parceiro.

Para 92\% $(n=46)$ dos participantes, o encaminhamento dos casos de mulheres maltratadas deve ser uma tarefa dos profissionais de saúde e $70 \% \quad(n=35)$ consideraram ainda que o enfermeiro pode ser 0 profissional mais adequado para encaminhar as vítimas. Dos que afirmaram já ter identificado uma mulher vítima de violência, 54\% ( $n=27)$ não respondeu sobre qual tipo de conduta que ofereceram à vítima e $62 \%$ (n=31) não respondeu para onde as encaminharam.

Dos enfermeiros de família participantes, $90 \%(n=45)$ são da opinião que as mulheres maltratadas não dizem respeito apenas ao setor jurídico, mas também ao da saúde. Um total de $98 \%(n=49)$ considerou que seria útil para os enfermeiros de família terem protocolos de deteção de casos de mulheres vítimas de violência pelos seus parceiros e $92 \% \quad(n=46)$ também consideraram útil registar os casos de mulheres agredidas identificadas no âmbito da sua consulta, mas 


\section{Violência doméstica contra as mulheres: conhecimentos, atitudes e barreiras do enfermeiro de família}

que esta prática deveria estar regulamentada. Relativamente às questões colocadas às vítimas, 76\% $(n=38)$ consideraram que estas devem ser incluídas como perguntas de rotina na avaliação inicial, embora $66 \%(n=33)$ tenha considerado que estas podem ofendê-las ou incomodá-las. Para 90\% (n=40) dos participantes, a prática clínica, em comparação com o apoio jurídico e psicológico, carece de importância em relação ao cuidado que pode ser prestado a uma mulher maltratada pelo seu parceiro. Dos participantes, $78 \%(n=39)$ consideraram ainda que quando um enfermeiro ajuda e/ou orienta uma mulher vítima de abuso, existe a possibilidade de este vir a sofrer represálias por parte do agressor.

A violência doméstica contra a mulher foi considerada pela totalidade dos participantes um tema interessante para se dedicar tempo e capacitação, sendo que $96 \%(n=48)$ é da opinião que existe uma necessidade de formação e que esta pode ser inserida nas especialidades de enfermagem e cursos de pósgraduação. Dos enfermeiros de família participantes, 92\% ( $n=46)$ demonstraram interesse em participar num curso ou formação sobre violência doméstica contra as mulheres, tendo em conta a necessidade de proporcionarem cuidados terapêuticos neste tipo de casos de violência.

\section{Barreiras}

As barreiras referem-se aos fatores institucionais e da prática clínica que dificultam a identificação e encaminhamento da mulher vítima de violência doméstica pelo seu parceiro.

Na opinião dos participantes, $36 \%(n=18)$ considera que os principais fatores institucionais que são percecionados como barreiras na identificação e encaminhamentos dos casos de mulheres agredidas que chegam à consulta são a falta de protocolos para detenção ou orientação dos casos; $28 \% \quad(n=14)$ consideram a falta de legitimação da participação do enfermeiro do ponto de vista legal e institucional e $14 \%(n=7)$ consideram o seu desconhecimento sobre o tema. Na prática clínica, 52\% $(n=26)$ consideraram como barreiras que dificultam a identificação das vítimas o caráter privado da violência, $12 \% \quad(n=6)$ consideraram a falta de conhecimento sobre o tema e $10 \%(n=5)$ a falta de tempo na consulta.

\section{DISCUSSÃO}

A maior parte dos participantes nunca teve formação sobre o tema no seu percurso académico, manifestando interesse neste tipo de formação. Resultados semelhantes foram descritos numa pesquisa realizada no Brasil sobre o rastreio e encaminhamento das mulheres vítimas de violência que apontou para a necessidade da qualificação da equipa de enfermagem (Lima et al., 2020). Num outro estudo sobre a conduta de enfermeiras perante casos de violência contra a mulher, a totalidade das entrevistadas sentiram necessidade de capacitação para lidar com as questões de violência doméstica contra mulher (Silva, Trindade, \& Lima, 2019). Numa revisão mais alargada sobre a compreensão que os enfermeiros têm sobre a violência doméstica contra as mulheres concluiu que aos enfermeiros não é proporcionada formação suficiente que Ihes permita lidar, reconhecer e identificar mulheres vítimas de violência, reforçando a necessidade de se apostar nesta área de formação desde a formação pré- 


\section{Violência doméstica contra as mulheres: conhecimentos, atitudes e barreiras do enfermeiro de família}

graduada (Alshammari, McGarry \& Higginbottom, 2018).

Os participantes consideraram que a mulher vítima de violência doméstica pode apresentar danos físicos, sexuais e psicológicos. Tais resultados são idênticos aos de um estudo recente onde o conceito de violência relatado pelas profissionais de enfermagem teve em consideração aspetos de natureza física, psicológica e social (Lima et al., 2020). Num outro estudo, realizado em Portugal, sobre a representação social da violência doméstica sobre as mulheres, os participantes associaram este tipo de violência a termos como o medo, o sofrimento e a dor (Oliveira, Figueiredo, Nina, Oliveira, \& Novais, 2019), indo ao encontro das caraterísticas psicológicas agora identificadas pelos participantes.

A maioria dos participantes considerou que o motivo de o homem praticar violência doméstica é o facto de este ter presenciado situações de violência na infância, no entanto, embora ocorram fenómenos de transmissão intergeracional da violência, apenas uma minoria dos agressores foi alguma vez vítima no passado (Centro de Estudos Judiciários, 2016).

No que respeita às atitudes afetivas de identificação das mulheres, os participantes consideram que numa consulta de rotina esta identificação deve fazer parte das tarefas do enfermeiro. No artigo sobre a Assistência de Enfermagem, mais especificamente, na narrativa das mulheres vítimas de violência doméstica, as vítimas afirmaram que os enfermeiros têm mais oportunidades para detetar estes casos (Cristina, Risso, \& Sim, 2019). No estudo sobre o rastreio e encaminhamento de casos de violência contra a mulher, os participantes consideraram que a consulta de enfermagem é o momento estratégico para identificar situações de mulheres vítimas de violência doméstica, devendo utilizar estratégias para esclarecimento da situação, a fim de traçar um plano de cuidado adequado, respeitando as necessidades da vítima (Lima et al., 2020).

Relativamente à frequência com que os participantes já identificaram mulheres maltratadas pelos seus parceiros, os dados encontrados são inferiores aos encontrados a nível internacional, em que pelo menos $60 \%$ dos enfermeiros refere ter já identificado mulheres vítimas de violência, ressaltando, no entanto a referência a baixas taxas de reporte dessas situações por parte dos profissionais (Cho, Cha \& Yoo, 2015).

A maioria dos participantes foram da opinião que o enfermeiro de família deve encaminhar as mulheres maltratadas, contudo, mais da metade não respondeu qual o tipo de conduta que ofereceram e nem para onde encaminharam estas mulheres. Num estudo semelhante, realizado em Israel, os enfermeiros especificam o tipo de conduta e encaminhamento que oferecem a estas mulheres, afirmando que as apoiaram, disponibilizaram informações sobre serviços que prestam assistência e aconselhamento, convocaram a assistente social, registaram as informações recebidas, agendaram acompanhamentos, entre outros (Natan \& Rais, 2010). Os enfermeiros de família consideraram que seria útil terem protocolos de deteção de casos de mulheres que foram vítimas de violência pelos seus parceiros indo ao encontro de evidência que ressalta a falta de apoio legal e ausência de protocolos que direcionem a atuação destes profissionais no atendimento à mulher em situação de violência e que tal dificultou a conduta 
a ser adotada perante os casos identificados (Silva, Trindade, \& Lima, 2019).

A maioria dos enfermeiros de família, do presente estudo, foi da opinião que existe uma necessidade em participar em cursos ou formações sobre violência doméstica contra a mulher, o que vai ao encontro de resultados de outros estudos que identificam a necessidade de capacitação dos profissionais para abordagem a estas mulheres (Silva, Trindade, \& Lima, 2019) e que estes se sentem insuficientemente preparados para identificar e estabelecer cuidados à mulher vítima de violência (Alshammari, McGarry \& Higginbottom, 2018; Rodrigues et al., 2018).

\section{CONCLUSÃO}

A maioria dos participantes refere não ter tido formação sobre esta temática e evidenciam falta de conhecimento sobre a mesma, reconhecendo, no entanto, que a identificação e encaminhamento de mulheres vítimas de violência doméstica é responsabilidade dos profissionais de saúde. Apontam como principais barreiras a falta de protocolos de atuação e regulamentação que legitime a sua intervenção e, em menor percentagem, a falta de conhecimento/formação para a abordagem á vítima.

Pela importância da problemática e da pertinência absoluta da sua integração na agenda política da saúde e da formação dos seus profissionais, designadamente dos enfermeiros, sugere-se a continuidade $e$ aprofundamento de estudos desta natureza. A investigação deve prosseguir no sentido de dimensionar as necessidades ao nível da formação pré e pós-graduada, infundindo nos currículos esta temática e capacitando assim atuais e futuros profissionais na identificação e abordagem à mulher vítima de violência doméstica. Poderá ainda ser considerado como perspetiva futura a validação deste instrumento para a população portuguesa.

Este estudo tem limitações, nomeadamente a dimensão da amostra, tipo de amostragem e o facto de ser constituída maioritariamente por mulheres, a realizar formação pós-graduada ou participar em eventos numa só instituição de ensino superior, factos que poderão enviesar os resultados dos seus conhecimentos e atitudes, devendo por isso os mesmos ser interpretados com cautela.

\section{REFERÊNCIAS BIBLIOGRÁFICAS}

Alshammari, K. F., McGarry, J., \& Higginbottom, G. (2018). Nurse education and understanding related to domestic violence and abuse against women: An integrative review of the literature. Nursing open, 5(3), 237-253. https://doi.org/10.1002/nop2.133

Associação Portuguesa de Apoio à Vítima. (2020). Estatísticas APAV. Relatório Anual 2019. Lisboa. Retirado de https://apav.pt/apav_v3/images/pdf/Estatisticas_APA V-Relatorio_Anual_2019.pdf

Centro de Estudos Judiciários. (2016). Violência Doméstica: Implicações psicológicas, sociológicas e jurídicas do fenómeno. Manual Pluri Disciplinar. Lisboa. Retirado de http://www.cej.mj.pt/cej/recursos/ebooks/outros/Vi olencia-Domestica-CEJ_p02_rev2c-

EBOOK_ver_final.pdf

Cho, O. H., Cha, K. S., \& Yoo, Y. S. (2015). Awareness and Attitudes Towards Violence and Abuse among Emergency Nurses. Asian nursing research, 9(3), 213218. https://doi.org/10.1016/j.anr.2015.03.003

Conselho Internacional dos Enfermeiros. (2016). CIPE ${ }^{\circledast}$ Versão 2015 - Classificação Internacional para a Prática de Enfermagem. Lisboa: Ordem dos Enfermeiros.

Cristina, I., Risso, S., \& Sim, M. (2019). Assistência de Enfermagem - Narrativa das mulheres vítima de violência doméstica. Revista Ibero Americana de Saúde 
e Envelhecimento, 5 (3), 1998-2014. doi: 10.24902/r.riase.2019.5(3).1998

Decreto-Lei n.o 118/2014, de 5 de agosto (2014). Diário da República n.o 149 - I série. Ministério da Saúde. Lisboa, Portugal.

Lei no 59/2007, de 4 de setembro (2007). Diário da República n. 170 - I série. Ministério da Justiça. Lisboa, Portugal.

Lei n. 156/2015, de 16 de setembro (2015). Diário da República no 181 - I Série. Ministério da Saúde. Lisboa, Portugal.

Lima, J., Santos, R., Silva, J., Silva, R., Souto, C., Souto, R., \& Araújo, G. (2020). Rastreio e encaminhamento de casos de violência contra a mulher por enfermeiras na estratégia saúde da família. Cogitare Enfermagem. doi:10.5380/ce.v25i0.65579

Méndez-Hernández, P., Valdez-Santiago, R., ViniegraVelázquez, L., Rivera-Rivera, L., \& Salmerón-Castro, J. (2003). Violencia contra la mujer: conocimiento y actitud del personal médico del Instituto Mexicano del Seguro Social, Morelos, México. Salud Pública México, 45 (6), 472-482. Retirado de http://www.scielo.org.mx/scielo.php?script=sci_artte $x t \& p i d=S 0036-36342003000600007$

Natan, M., \& Rais, I. (2010). Knowledge and Attitudes of Nurses Regarding Domestic Violence and Their Effect on the Identification of Battered Women. Journal of Trauma Nursing, 17, 112-117. doi:10.1097/JTN.0b013e3181e736db

Oliveira, I., Figueiredo, B., Nina, J., Oliveira, X., \& Novais, S. (2019). Representação social da violência doméstica sobre as mulheres. Revista de Investigação \& Inovação em Saúde, 2 (2), 7-18. doi:10.37914/riis.v2i2.53
Organização Mundial da Saúde. (2020). COVID-19 e a violência contra a mulher. $O$ que o setor/sistema de saúde pode fazer. Retirado de https://www.who.int/reproductivehealth/publication s/vaw-covid-19/pt/

Presidência do Conselho de Ministros no 103/2013. (2013). V Plano Nacional de Prevenção e Combate à Violência Doméstica e de Género 2014-2017. Diário da República: I série, no 253. 7036-7049. Retirado de https://dre.pt/application/conteudo/136785

Regulamento n. 428/2018, de 16 de julho (2018). Diário da República no 135 - Il Série. Ordem dos Enfermeiros. Lisboa, Portugal.

Rodrigues, V., Oliveira, G., Machado, J., Simões, A., Pires, V., \& Morais, R. (2018). Assistência à Saúde da Mulher em Situação de Violência Doméstica: Revisão Integrativa. Revista Saúde Com, 14 (1), 1122-1129. doi: 10.22481/rsc.v14i1.538

Silva, P., Trindade, R., \& Lima, W. (2019). Conduta de enfermeiras diante de casos de violência doméstica contra a mulher. Revista Baiana de Enfermagem, 33, e33452 1-11. doi: 10.18471/rbe.v33.33452

Wild, D., Grove, A., Martin, M., Eremenco, S., McElroy, S., Verjee-Lorenz, A., \& Erikson, P. (2005). Principles of Good Practice for the Translation and Cultural Adaptation Process for Patients-Reported Outcomes (PRO) Measures: Report for ISPOR Task Force for Translation and Cultural Adaptation. Value in Health, 8 (2), 94-104. Retirado de https://onlinelibrary.wiley.com/doi/epdf/10.1111/j.1 524-4733.2005.04054.x

World Health Organization. (2017). Violence against women: Intimate partner and sexual violence against women. Retirado de http://www.who.int/mediacentre/factsheets/fs239/e $\mathrm{n} /$ 